\title{
Uso de Diferentes Herbicidas no Controle de Myriophyllum aquaticum ${ }^{1}$
}

\author{
Chemical Control of Myriophyllum aquaticum
}

\begin{abstract}
NEGRISOLI, E. ${ }^{2}$, TOFOLI, G.R. ${ }^{2}$, VELINI, E.D. ${ }^{3}$, MARTINS, D. ${ }^{3}$ e CAVENAGHI, A.L. ${ }^{2}$
RESUMO - Este estudo teve a finalidade de avaliar, em condições de caixa d'água, o controle químico de Myriophyllum aquaticum (pinheiro-d'água), através de herbicidas aplicados em pós-emergência. Os herbicidas e respectivas doses $\left(\mathrm{g} \mathrm{ha}^{-1}\right)$ foram: diquat (Reward) a 204 g i.a. ha-1 ${ }^{-1}$ diquat a 102 e 204 g i.a. ha-1 + Agral a 0,1\%; 2,4-D (DMA 806 BR) a 167, 335, 670 e 1.340 g e.a. ha ${ }^{-1}$; glyphosate (Rodeo) a 3.360 g e.a. ha-1 + Aterbane a 0,5\%; e imazapyr (Arsenal) a $250 \mathrm{~g}$ e.a. ha $^{-1}$. As parcelas foram constituídas por caixas d'água de 0,60 x 0,60 x $0,45 \mathrm{~m}$, com 120 litros de água +20 litros de solo e 20 ramos por caixa. Utilizou-se um pulverizador costal a pressão constante de $\mathrm{CO}_{2}$ a 2 bar, pontas $110.02 \mathrm{VS}$, com um consumo de calda de $1801 \mathrm{ha}^{-1}$. O controle foi avaliado visualmente aos 2, 6, 9,11, 13, 17, 20, 23, 26, 30 e 36 dias após a aplicação dos herbicidas (DAAH). Inicialmente, o herbicida diquat foi o composto que apresentou os sintomas mais severos de intoxicação nos ramos de pinheirod'água aos 2 DAAH, com 65\% de controle em média, e aos 20 DAAH ele atingiu o controle máximo (99\%), porém ocorreram rebrotas a partir dos 23 DAAH, independentemente da adição ou não de Agral e das doses testadas. O herbicida 2,4-D proporcionou $100 \%$ de controle dos ramos a partir dos 23 DAAH para as doses de 1.340 e $670 \mathrm{~g} \mathrm{ha}^{-1}$, não ocorrendo rebrotas; já para as demais doses testadas (335 e $167 \mathrm{~g} \mathrm{ha}^{-1}$ ) o controle não foi eficiente, pois ocorreram rebrotas. Os herbicidas glyphosate e imazapyr não foram eficientes no controle desta espécie.
\end{abstract}

Palavras-chave: planta aquática, 2,4-D, diquat, glyphosate, imazapyr.

\begin{abstract}
This work was carried out at the Advanced Weed Research Nucleus - UNESP, Botucatu-SP, to evaluate the control of Myriophyllum aquaticum by applying different herbicides in post emergence. The herbicides and doses ( $\left.\mathrm{ha}^{-1}\right)$ tested were: diquat (Reward) at $204 \mathrm{~g}$ a.i. $\mathrm{ha}^{-1}$, diquat at 102 and $204 \mathrm{~g}$ a.i. $\mathrm{ha}^{-1}+$ agral at $0.1 \% \mathrm{v} / \mathrm{v} ; 2,4-\mathrm{D}$ (DMA $806 \mathrm{BR}$ ) at 1,340, 670, 335 and $167 \mathrm{~g}$ a.e. ha-1 glyphosate (Rodeo) at $3.360 \mathrm{~g}$ a.e. $\mathrm{ha}^{-1}+$ Aterbane at $0.5 \% \mathrm{v} / \mathrm{v}$ and imazapyr (Arsenal) at $250 \mathrm{~g}$ a.e. $\mathrm{ha}^{-1}$. Small tanks $(60 \times 60 \times 45 \mathrm{~cm})$ with about $120 \mathrm{~L}$ water capacity, plus $20 \mathrm{~L}$ of soil were used. The plants were counted (20 stems), and distributed in the plots. Plant phytotoxicity was evaluated at 2, 6, 9, 11, 13, 17, 20, 23, 26, 30 and 36 days after application (DAA). Herbicide spraying was performed with a backpack sprayer, using a 2.0 bar for $\mathrm{CO}_{2}$ pressure, consuming $180 \mathrm{l} \mathrm{ha} \mathrm{h}^{-1}$ volume and $110.02 \mathrm{VS}$ fan type nozzles. The herbicide diquat showed the first symptoms of intoxication at 2 DAA, and regardless of the dose applied, provided overall control of Myriophyllum plants at 20 DAA, with plant regrowth being observed at 23 DAA. The 2,4 D herbicide provided 100\% control at 23 DAA, for doses 1,340 and $670 \mathrm{~g} \mathrm{ha}^{-1}$. However, for the other doses tested (335 and $167 \mathrm{~g} \mathrm{ha}^{-1}$ ), the control was not effective. The herbicides glyphosate and imazapyr were not efficient in controlling this plant.
\end{abstract}

Key words: aquatic weed, 2,4 D, diquat, glyphosate, imazapyr.

Recebido para publicação 28.8.2002 e na forma revisada em 5.11.2002.

2 Eng.-Agr. M.S., aluno de Pós-Graduação em Agricultura, nível de Doutorado, Departamento de Produção Vegetal da Faculdade de Ciências Agronômicas - FCA-UNESP, Caixa Postal 237, 18603-970 Botucatu-SP, Brasil, <ednegri@fca.unesp.br> ${ }^{3}$ Prof. Dr., Departamento de Produção Vegetal da FCA-UNESP.

Planta Daninha, Viçosa-MG, v.21, p.89-92, 2003. Edição Especial 


\section{INTRODUÇÃO}

A proliferação de plantas infestantes aquáticas constitui-se hoje em um sério e complexo problema, de repercussão não apenas em nivel ambiental, pois atinge igualmente as áreas econômicas e sociais, como: pesca, lazer, navegação e atividades ligadas à agropecuária (Marcondes \& Tanaka, 1997).

Uma grande diversidade de plantas aquáticas pode ser encontrada vegetando as margens de rios e reservatórios ou dentro dos mais diversos ambientes aquáticos, empregando diferentes mecanismos de adaptação para sobrevivência e desenvolvimento. Enquanto algumas espécies apresentam-se enraizadas em corpos d'água com fortes correntezas, outras somente podem viver em águas paradas ou estagnadas (Martins et al., 2002).

Myriophyllum brasiliense (Camb) é sinônimo de Myriophyllum aquaticum (Vell.) Verdc, planta aquática muito difundida mundialmente, pertencente à classe Dicotiledoneae, ordem Myrtales, família Haloragaceae (Couch \& Nelson, 1991), sendo originária da América do Sul e de ocorrência natural no Brasil. É uma planta perene, aquática, podendo se desenvolver totalmente submersa, como nos aquários, ou com a porção terminal dos ramos emersa. A planta mantém-se enraizada no lodo, no fundo de lagos com até $2 \mathrm{~m}$ de profundidade, ou nas margens, deixando que os ramos avancem pela água; a parte terminal dos ramos geralmente emerge em concentrações densas, que permitem apoio - a parte fora da água pode atingir $30 \mathrm{~cm}$ de comprimento (Kissmann, 2000). Apesar de ser de água doce, é uma das plantas aquáticas que toleram a maior concentração de salinidade; só a partir de concentrações de $1 \%$ de cloreto de sódio na água começa a se inibir o desenvolvimento.

O controle mecânico é um método de baixo custo e, na maioria das situações, eficiente no controle de plantas aquáticas (MacDonald \& Langeland, 2001), exceto em situações especiais, como é o caso do Myriophyllum aquaticum, que se reproduz por sementes; contudo, sua disseminação é feita principalmente por fragmentos ou pequenas estruturas das plantas, o que compromete a possibilidade do uso de técnicas de controle mecânico, sob pena de aumentar a população infestante quando utilizado este método de controle (Reynolds, 1999).

Resultados satisfatórios no controle químico de Myriophyllum aquaticum foram observados por MacDonald \& Langeland (2001), mostrando que o herbicida 2,4-D apresentou excelente controle desta planta. Portanto, o controle químico, para esta espécie, torna-se uma alternativa satisfatória.

Embora o Myriophyllum seja considerado por muitos como suscetível a vários herbicidas, é difícil obter o controle completo da infestante (Washington Departament of Ecology Water Quality Program, 1998). Assim, o objetivo deste trabalho foi o de avaliar diferentes herbicidas e doses no controle de plantas de Myriophyllum aquaticum em condições de caixa d'água.

\section{MATERIAL E MÉTODOS}

O experimento foi instalado na Faculdade de Ciências Agronômicas/UNESP, campus de Botucatu-SP, no núcleo de Pesquisas Avançadas em Matologia.

As unidades experimentais foram constituídas por caixa de fibra de vidro, com plantas, contendo as seguintes dimensões: 0,6 x 0,6 x $0,45 \mathrm{~m}$ com 120 litros de água. Foi colocada em cada caixa uma camada de solo de 20 litros. As características químicas do solo utilizado nos diferentes experimentos foram: $\mathrm{pH}\left(\mathrm{CaCl}_{2}\right)$ $=5,5 ;$ M.O. $\left(\mathrm{g} \mathrm{dm}^{-3}\right)=23 ; \mathrm{P}, \mathrm{Cu}, \mathrm{Fe}, \mathrm{Mn}$ e $\mathrm{Zn}=$ 9; 0,$12 ; 6,10 ; 34,6$; e $1,1 \mathrm{mg} \mathrm{dm}^{-3}$, respectivamente; $\mathrm{H}+\mathrm{Al}, \mathrm{K}, \mathrm{Ca}, \mathrm{Mg}, \mathrm{SB}$ e CTC $=55 ; 3,3$; 17; 6; 24; e $77 \mathrm{mmol}_{\mathrm{c}} \mathrm{dm}^{-3}$, respectivamente; e $\mathrm{V} \%=33$. As características físicas do solo eram: $57 \%$ de argila, $37 \%$ de areia e $6 \%$ de silte. Cada caixa foi adubada com $40 \mathrm{~g}$ da fórmula 4-14-8 de NPK.

As caixas d'água foram mantidas em condições de pleno sol, e utilizaram-se 15 ramos/ caixa de M. aquaticum, as quais foram coletadas em área pertencente à Fazenda Experimental Lageado-Unesp Botucatu, sendo as plantas distribuídas uniformemente nas caixas d'água. Os tratamentos foram dispostos no delineamento experimental em blocos ao acaso, com quatro repetições.

Os herbicidas e doses testados foram: diquat (Reward) a 204 g i.a. ha-1 diquat a 102 e 204 g i.a. ha-1 + Agral a 0,1\%; 2,4-D (DMA 
$806 \mathrm{BR})$ a $167,335,670$ e $1.340 \mathrm{~g}$ e.a ha ${ }^{-1}$; glyphosate (Rodeo) a 3.360 g e.a. ha ${ }^{-1}+$ Aterbane a 0,5\%; imazapyr (Arsenal) a 250 g e.a. ha ${ }^{-1}$; e, ainda, uma testemunha sem aplicação de herbicidas. Os herbicidas foram aplicados em plantas adultas.

A aplicação dos herbicidas foi feita com um pulverizador costal com pressão constante de $\mathrm{CO}_{2}$ de 2,0 bar. O consumo de calda utilizado foi de $180 \mathrm{~L} \mathrm{ha}^{-1}$. A barra de aplicação apresentava-se com duas pontas de pulverização do tipo jato plano, TeeJet $110.02 \mathrm{VS}$, distanciadas entre si de $0,5 \mathrm{~m}$.

As condições ambientais durante a aplicação dos herbicidas foram divididas em condição inicial, intermediária e final, durante o tempo em que se realizou a aplicação dos herbicidas, que foi de 50 minutos. A temperatura foi de 25,26 e $25^{\circ} \mathrm{C}$; a umidade relativa, de 65,66 e $66 \%$; e a velocidade do vento, de 2,0, 2,0 e 3,0 $\mathrm{m} \mathrm{s}^{-1}$, respectivamente.

As plantas de pinheirinho-d'água foram avaliadas visualmente aos $2,6,9,11,13,17$, $20,23,26,30$ e 36 dias após a aplicação dos herbicidas (DAAH), por meio de uma escala percentual de notas, em que zero consistia em nenhum controle e $100 \%$ em controle total das plantas. As avaliações foram realizadas até o desaparecimento ou a estabilização dos sintomas de fitointoxicação (SBCPD, 1995).
Os parâmetros utilizados no estabelecimento das notas foram: quantidade de plantas mortas; acúmulo de biomassa; inibição do crescimento; quantidade e uniformidade das injúrias; e capacidade de rebrota das plantas.

Os resultados obtidos foram submetidos à análise de variância pelo teste $\mathrm{F}$, e as médias dos tratamentos foram comparadas pelo teste $\mathrm{T}$ a $5 \%$ de probabilidade.

\section{RESULTADOS E DISCUSSÃO}

Inicialmente, o herbicida diquat foi o composto que proporcionou os sintomas mais severos de intoxicação às folhas e aos ramos do pinheirinho-d'água, promovendo queimaduras severas que evoluíram para necroses e, em seguida, para uma aparente morte das plantas. Já para o herbicida glyphosate, os sintomas visuais e iniciais foram o amarelecimento de folhas e ramos, seguido de queimaduras e necroses. No caso do $2,4-\mathrm{D}$, o sintoma inicial foi encarquilhamento e tombamento dos ramos, havendo posteriormente o apodrecimento e a morte da planta. O herbicida imazapyr proporcionou poucos sintomas iniciais de injúrias às plantas desta espécie.

Na Tabela 1 estão apresentados os resultados da avaliação de controle sobre as plantas de $M$. aquaticum em diferentes épocas após a

Tabela 1 - Porcentagem de controle de Miriophylum aquaticum em diferentes períodos de avaliação após a aplicação de diversos herbicidas. Botucatu-SP, 1999

\begin{tabular}{|c|c|c|c|c|c|c|c|c|c|c|c|c|}
\hline \multicolumn{13}{|c|}{ Dias após a aplicação } \\
\hline Tratamento & $\begin{array}{c}\text { Dose } \\
\left(\mathrm{g} \mathrm{e} . \mathrm{a} \mathrm{ha}^{-1}\right)\end{array}$ & 2 & 6 & 9 & 11 & 13 & 17 & 20 & 23 & 26 & 30 & 36 \\
\hline 1. diquat $^{1 /}$ & 204 & $62,50 \mathrm{a}$ & $80,00 \mathrm{~b}$ & $95,25 \mathrm{a}$ & $95,75 \mathrm{a}$ & $95,75 \mathrm{a}$ & $98,75 \mathrm{a}$ & $98,75 \mathrm{a}$ & $68,75 \mathrm{c}$ & $70,00 \mathrm{~d}$ & $60,00 \mathrm{c}$ & $52,50 \mathrm{c}$ \\
\hline 2. diquat $^{2 /}$ & 102 & $66,25 \mathrm{a}$ & $90,50 \mathrm{a}$ & $97,50 \mathrm{a}$ & $97,75 \mathrm{a}$ & $97,75 \mathrm{a}$ & $99,00 \mathrm{a}$ & $99,00 \mathrm{a}$ & $72,50 \mathrm{c}$ & $75,00 \mathrm{c}$ & $60,00 \mathrm{c}$ & $53,75 \mathrm{c}$ \\
\hline 3. diquat $^{2 /}$ & 204 & $65,00 \mathrm{a}$ & $94,00 \mathrm{a}$ & $96,75 \mathrm{a}$ & $97,50 \mathrm{a}$ & $97,50 \mathrm{a}$ & $99,00 \mathrm{a}$ & $99,00 \mathrm{a}$ & $68,75 \mathrm{c}$ & $70,00 \mathrm{~d}$ & $60,00 \mathrm{c}$ & $52,50 \mathrm{c}$ \\
\hline 4. $2,4-\mathrm{D}$ & 167 & $2,00 \mathrm{c}$ & $42,50 \mathrm{~d}$ & $50,00 \mathrm{c}$ & $45,00 \mathrm{~d}$ & $45,00 \mathrm{~d}$ & $38,75 \mathrm{c}$ & $26,25 \mathrm{~b}$ & $20,00 \mathrm{e}$ & $6,00 \mathrm{f}$ & $4,25 \mathrm{e}$ & $4,25 \mathrm{e}$ \\
\hline 5. $2,4-\mathrm{D}$ & 335 & $3,75 \mathrm{cb}$ & $68,75 \mathrm{c}$ & $75,00 \mathrm{~b}$ & $80,00 \mathrm{~b}$ & $81,25 \mathrm{~b}$ & $92,75 \mathrm{~b}$ & $97,00 \mathrm{a}$ & $98,00 \mathrm{a}$ & $98,00 \mathrm{a}$ & $82,50 \mathrm{~b}$ & $60,00 \mathrm{~b}$ \\
\hline 6. $2,4-\mathrm{D}$ & 670 & $8,50 \mathrm{~b}$ & $72,50 \mathrm{c}$ & $77,50 \mathrm{~b}$ & $82,50 \mathrm{~b}$ & $81,25 \mathrm{~b}$ & $95,75 a b$ & $99,00 \mathrm{a}$ & $99,00 \mathrm{a}$ & $100,00 \mathrm{a}$ & $100,00 \mathrm{a}$ & $100,00 \mathrm{a}$ \\
\hline 7. $2,4-\mathrm{D}$ & 1.340 & $7,75 \mathrm{~b}$ & $71,25 \mathrm{c}$ & $78,00 \mathrm{~b}$ & $81,25 \mathrm{~b}$ & $81,25 \mathrm{~b}$ & $99,00 \mathrm{a}$ & $99,00 \mathrm{a}$ & $99,00 \mathrm{a}$ & $100,00 \mathrm{a}$ & $100,00 \mathrm{a}$ & $100,00 \mathrm{a}$ \\
\hline 8. glyphosate ${ }^{3 /}$ & 3.360 & $4,25 \mathrm{cb}$ & $5,75 \mathrm{e}$ & $73,25 \mathrm{~b}$ & $72,50 \mathrm{c}$ & $72,50 \mathrm{c}$ & $97,50 \mathrm{ab}$ & $98,50 \mathrm{a}$ & $93,00 \mathrm{~b}$ & $81,50 \mathrm{~b}$ & $60,00 \mathrm{c}$ & $33,75 \mathrm{~d}$ \\
\hline 9. imazapyr & 250 & $1,50 \mathrm{c}$ & $3,00 \mathrm{e}$ & $3,25 \mathrm{~d}$ & $4,00 \mathrm{e}$ & $5,00 \mathrm{e}$ & $27,50 \mathrm{~d}$ & $27,50 \mathrm{~b}$ & $62,50 \mathrm{~d}$ & $20,00 \mathrm{e}$ & $16,25 \mathrm{~d}$ & $8,50 \mathrm{e}$ \\
\hline 10. testemunha & - & - & - & - & - & - & - & - & - & - & - & - \\
\hline F tratamento & & $328,11 * *$ & $471,12^{* *}$ & $243,72 * *$ & $742,44 * *$ & $753,11^{* *}$ & $202,00 * *$ & $269,81 * *$ & $382,08 * *$ & $766,62 * *$ & $489,77 * *$ & $446,36^{* *}$ \\
\hline F bloco & & $0,10^{\mathrm{ns}}$ & $2,75^{\mathrm{ns}}$ & $0,23^{\mathrm{ns}}$ & $0,15^{\mathrm{ns}}$ & $0,46^{\mathrm{ns}}$ & $0,24^{\mathrm{ns}}$ & $0,88^{\mathrm{ns}}$ & $1,17^{\mathrm{ns}}$ & $2,90^{\mathrm{ns}}$ & $1,22^{\mathrm{ns}}$ & $0,66^{\mathrm{ns}}$ \\
\hline $\mathrm{CV}(\%)$ & & 13,5 & 5,4 & 5,3 & 0,8 & 3,0 & 4,8 & 4,7 & 3,5 & 3,6 & 5,0 & 5,9 \\
\hline D. m. s. & & 4,847 & 4,592 & 5,566 & 3,276 & 3,222 & 5,863 & 5,622 & 3,831 & 3,599 & 4,356 & 4,483 \\
\hline
\end{tabular}

${ }^{1 /}$ Dose em i.a ha ${ }^{-1} .{ }^{2 /}$ Adicionou-se Agral a $0,1 \%$. ${ }^{3 /}$ Adicionou-se Aterbane a $0,5 \%$.

Médias seguidas de mesma letra, na coluna, não diferem estatisticamente entre si pelo teste $t(P>0,05)$. ns - não-significativo e $* *$ - significativo a $1 \%$ de probabilidade. 
aplicação dos herbicidas (DAAH). As parcelas tratadas com o herbicida diquat, aos 2 DAAH, apresentaram inicialmente controle elevado, que evoluiu e atingiu aos 20 DAAH máximo controle, porém ocorreram rebrotas a partir dos 23 DAAH, independentemente da adição ou não de Agral e das doses aplicadas, o que evidenciou um controle não-satisfatório do diquat sobre esta espécie.

Guanto ao herbicida 2,4-D, a aplicação da dose de $167 \mathrm{~g} \mathrm{ha}^{-1}$ não proporcionou controle satisfatório, visto que ocorreram rebrotas a partir de 17 DAAH. Já para a dose de $335 \mathrm{~g} \mathrm{ha}^{-1}$, observa-se aos 26 DAAH um excelente controle, porém, aos $30 \mathrm{DAAH}$, ocorreram rebrotas, evidenciando um controle insatisfatório também para essa dose. Contudo, as doses de 670 e $1.340 \mathrm{~g} \mathrm{ha}^{-1}$ determinaram um controle total dos ramos a partir dos $26 \mathrm{DAAH}$ e não ocorreram rebrotas, evidenciando um efetivo e eficiente controle proporcionado por essas doses.

Resultados similares foram encontrados por MacDonald \& Langeland (2001), em que o herbicida 2,4-D apresentou um excelente controle de Myriophyllum spp. Também em um outro estudo no qual se trabalhou com a resposta de diferentes doses do herbicida 2,4 - D (ester), 4,4 e 8,9 $\mathrm{kg} \mathrm{ha}^{-1}$, foram obtidas respostas positivas, ou seja, eficientes, quando aplicados em plantas jovens, ainda em crescimento, e em folhas emergentes (Washington Department of Ecology Water Quality Program, 1998).

O herbicida glyphosate mostrou-se eficaz no controle de $M$. aquaticum aos 17 DAAH; entretanto, após este período ocorreram rebrotas, o que reduziu o seu controle, evidenciando a ineficácia do produto.

O herbicida imazapyr não apresentou controle satisfatório em nenhuma das épocas de avaliação. Aos 23 DAAH o imazapyr apresentou o seu máximo controle, porém, a partir dos 26 DAAH, ocorreram também rebrotas de plantas, o que inviabilizou a sua eficiência.

\section{LITERATURA CITADA}

COUCH, R.; NELSON, E. The exotic Myriophyllums of North America. Proceedings from the states' management programs-monitoring and lake assessments, 1991. p. 5-11.

KISSMANN, G. K. Plantas infestantes e nocivas. São Paulo: BASF, 2000. 608 p.

MacDONALD, E. G.; LANGELAND, A. K. Aquatic weed management alternatives for tropical areas. In:

CONGRESSO DE LA ASOCICION

LATINOAMERICANA DE MALEZAS, 15., 2001,

Maracaibo. Anais... Maracaibo: 2001. p. 44-47.

MARCONDES, D. A. S.; TANAKA, R. H. Plantas aquáticas nos reservatórios das usinas hidrelétricas da CESP. In: CONGRESSO BRASILEIRO DA CIÊNCIA DAS PLANTAS DANINHAS, 21., 1997, Caxambu. Workshop de Plantas Aquáticas... Caxambu: SBCPD, 1997. p. 2-4.

MARTINS, D. et al. Controle químico de Pistia stratiotes, Euchhornia crassipes e Salvinia molesta em caixas d'água. Planta Daninha, v. 20, p. 83-88, 2002.

REYNOLDS, B. Invasive alien plant species of Virginia. Course requirements at Virginia Polytechnic Institute \& State University, 1999. Disponível em: <http:// www.hort.vt.edu/vnps/>. Acesso em 28/08/2002.

SOCIEDADE BRASILEIRA DA CIÊNCIA DAS PLANTAS DANINHAS - SBCPD. Procedimentos para instalação, avaliação e análise de experimentos com herbicidas. Londrina: $1995.42 \mathrm{p}$.

WASHINGTON - DEPARTAMENT OF ECOLOGY WATER QUALITY PROGRAM. Technical information about Parrotfeather (Myriophyllum aquaticum).

Disponível em: <http://www.ecy.ma.gov/programs/wq/ plants/weeds/aqua003.html>. Acesso em 28/08/2002. 